\title{
Effect of intense pulsed-light therapy on hair regrowth in C57BL/6J mice mediated by WNT/ $\beta$-catenin signaling pathway
}

\author{
Lewen Jiang ${ }^{1,2}$, Yong Miao', Zhexiang Fan ${ }^{1}$, Jin Wang ${ }^{1}$, Zhiqi Hu ${ }^{1 *}$ \\ ${ }^{1}$ Department of Plastic and Aesthetic Surgery, Nanfang Hospital, Southern Medical University, Guangzhou 51000, China; \\ ${ }^{2}$ Department of Plastic and Aesthetic Surgery, Maternity \& Child Healthcare Hospital, Shenzhen 518116, China \\ *For correspondence: Email: nfzhiqihu@126.com; Tel: +86-020-61641862
}

Sent for review: 21 December 2017

Revised accepted: 26 April 2018

\begin{abstract}
Purpose: To evaluate the effect of low-fluence intense pulsed light (IPL) on hair growth in C57BL/6 mice, and to explore the potential molecular mechanisms of IPL actions on hair growth.

Methods: After low-fluence IPL irradiation was applied to depilated dorsal skin of C57BL/6 mice in the telogen, or resting hair cycle phase, tissue samples were obtained and used for histopathological analysis. Hair growth was analyzed by measuring hair length. In addition, protein expression levels of WNT3A and $\beta$-catenin were assayed by western blot.

Results: Low-fluence IPL irradiation promoted hair growth by inducing the anagen, or growth, phase in telogenic C57BL/6J mice. In particular, hair growth analysis suggested that application of low-fluence $I P L$ induced an earlier transition from telogen to anagen phase and prolonged the duration of anagen phase compared to the control group $(p<0.05)$. Moreover, western blotting assay revealed that WNT3A and $\beta$-catenin protein levels were up-regulated compared to the control group $(p<0.05)$.

Conclusion: These findings suggest that low-fluence IPL irradiation may be effective for promoting hair regrowth via activation of the WNT/ $\beta$-catenin pathway, and may, therefore, be a potential novel therapeutic treatment to stimulate hair regrowth.
\end{abstract}

Keywords: Intense pulsed light, Hair follicles, Hair growth, WNT3a/ß-catenin pathway

\begin{abstract}
This is an Open Access article that uses a funding model which does not charge readers or their institutions for access and distributed under the terms of the Creative Commons Attribution License (http://creativecommons.org/licenses/by/4.0) and the Budapest Open Access Initiative (http://www.budapestopenaccessinitiative.org/read), which permit unrestricted use, distribution, and reproduction in any medium, provided the original work is properly credited.
\end{abstract}

Tropical Journal of Pharmaceutical Research is indexed by Science Citation Index (SciSearch), Scopus, International Pharmaceutical Abstract, Chemical Abstracts, Embase, Index Copernicus, EBSCO, African Index Medicus, JournalSeek, Journal Citation Reports/Science Edition, Directory of Open Access Journals (DOAJ), African Journal Online, Bioline International, Open-J-Gate and Pharmacy Abstracts

\section{INTRODUCTION}

In recent years, the number of patients suffering from androgenic alopecia has increased dramatically, affecting $50 \%$ of adults over the age of 50 [1]. Although two current treatments, finasteride and minoxidil, have been approved by the United States Food and Drug Administration (FDA), their efficacy is limited and transient, due to adverse side effects and unpredictable efficacies [1]. Hair transplantation is a reliable surgical treatment for serious hair loss; however, its usefulness is limited by financial burden. Thus, the development of new therapies for hair loss is essential.

Paradoxical hypertrichosis, or unexpected hair growth, is a rare and significant side effect of intense pulsed light (IPL) and laser therapy. This phenomenon was first described in 2002 and has 
been widely acknowledged [2,3]. The incidence of paradoxical hypertrichosis ranges from $0.6 \%$ to $10 \%$. Patients with darker skin types (Fitzpatrick skin types III-VI) may be at increased risk for developing paradoxical hypertrichosis [2]. Paradoxical hypertrichosis is thought to be caused by sub-optimal laser fluencies that, are too low to induce thermolysis, but high enough to stimulate hair growth [4]. Although it may not be sufficient to epilate the hair, thermal energy at low-fluences can cause perifollicular inflammatory reactions for up to 2 weeks after treatment [2,5]. Vascular endothelial growth factor (VEGF) can be produced and secreted promptly when cells, such as vascular endothelial cells, keratinocytes and fibroblasts, are stimulated, and is involved in many processes, including inflammation, injury healing, and carcinogenesis [6].

Induction of angiogenesis is required to meet the increased nutritional needs of hair follicles during the anagen phase [7]. As a potent angiogenic growth factor, upregulation of VEGF is correlated with perifollicular angiogenesis [7]. These studies suggest that low-fluence IPL may help to induce hair growth through up-regulation of VEGF. However, the effects of low-fluence IPL on hair growth remain unclear.

Therefore, the present study investigated the effect of low-fluence IPL on hair growth and the underlying mechanism in mice. Results from these studies provide valuable insight for the development of new alternative therapies for hair loss.

\section{EXPERIMENTAL}

\section{Animal preparations}

Six-week-old female C57BL/6J mice were supplied by the Experimental Animal Center of Southern Medical University of China. All animal experiments were performed in accordance with the "Principles of Laboratory Animal Care" [8] and approved by the Experimental Animal Ethical Committee of Southern Hospital (approval no. 201701-S10) Southern Medical University of China (Guangzhou, China). All mice were housed in a standard environment $\left(25^{\circ} \mathrm{C}\right.$ and 60 $\%$ relative humidity) and provided with standard laboratory diet and water. Mice were allowed an acclimatization period of 1 week. The anagen phase model was induced by depilation of the dorsal skin of mice in telogen phase, as previously described [9]. A $12-\mathrm{cm}^{2}$ area $(3 \times 4$ $\mathrm{cm}$ ) of hair was removed by topical application of calcium thioglycolate.

\section{IPL treatment}

Fifty mice were divided into five randomized groups $(n=10)$ and used to study the effect of low-fluence IPL on hair growth. Cooled ultrasonic gel was applied to the depilated skin of mice in the low, medium and high groups and then the depilated area was irradiated using a $560 \mathrm{~nm}$ IPL (eCO2, Lutronic, Goyang, Korea). The fluence settings of the three IPL treatments were adjusted to 5,10 , and $15 \mathrm{~J} / \mathrm{cm}^{2}$ with a constant 3 $\mathrm{ms}$ pulse width, $10 \mathrm{~ms}$ delay time. Each irradiation was a $3 \mathrm{~cm}^{2}$ area $(1 \times 3 \mathrm{~cm})$ at a constant distance $(2 \mathrm{~mm})$ above each mouse. Mice in group 4 received no treatment (control), and group 5 received $200 \mu \mathrm{L} 2 \%$ minoxidil per day (positive). After IPL irradiation, mice were kept in isolation for $24 \mathrm{~h}$ and then rehoused in separate cages. Hair growth was evaluated using photography at $0,7,14$, and 21 days after treatment.

\section{Determination of hair growth}

Hair growth was evaluated using photography at 14 and 21 days. In addition, the hair length was measured to assess hair growth. Thirty hairs from each mouse were collected from treated dorsal areas by tweezers on day 14 and 21 . The lengths of hairs were measured by Vernier Calipers, and the average hair length per mouse was calculated.

\section{Histopathology of skin tissues}

Mice were sacrificed by cervical dislocation to obtain skin tissue specimens at day 21 posttreatment. Dorsal skin specimens were processed for hematoxylin and eosin ( $H$ \& $E$ ) staining. Fifty hair follicles per section were evaluated and categorized by hair cycle phases (telogen or anagen $\mathrm{I}-\mathrm{VI}$ ) based on the classifications of Chase [9]. Histopathological examinations were carried out under a microscope.

\section{Western blotting assays}

Dorsal skins tissue was harvested from mice for western blotting assays. Total protein was extracted and separated by odium dodecyl sulfate-polyacrylamide gel electrophoresis (SDSPAGE). Subsequently, proteins were probed with anti- $\beta$-catenin and anti-WNT3A (wingless-type MMTV integration site family member $3 a$ ) antibodies (Abcam, Cambridge, MA, USA). Protein bands were visualized by chemiluminescence detection, and protein expression levels were expressed relative to 
glyceraldehyde-3-phosphate (GAPDH).

dehydrogenase

\section{Statistical analysis}

Data were expressed as the mean \pm standard deviation (SD). Comparisons between groups were analyzed using Student's $t$-test or one-way analysis of variance (ANOVA) and Dunnett's $t$ test with SPSS version 18.0 (SPSS Inc., Chicago, IL, USA).

\section{RESULTS}

\section{Low-fluence IPL irradiation promotes hair growth}

Seven days after depilation, the dorsal skin color of the IPL treated groups was darker than the control group. By day 14, the dorsal skin hair recovery in IPL treated groups was enhanced compared to the control group. Dorsal skin hair was fully recovered by day 21 in the IPL treated and the positive group, but not in the control group (Figure 1). The average hair length in IPLtreated groups was significantly longer than that of the control group at both 14 and 21 days after depilation $(p<0.01)$ (Figure 2).

These results suggest that low-fluence IPL irradiation may induce hair growth by promoting early telogen-to-anagen conversion of hair follicles in C57BL/6J mice.

\section{Control Positive Low dose Middle dose High dose}

A
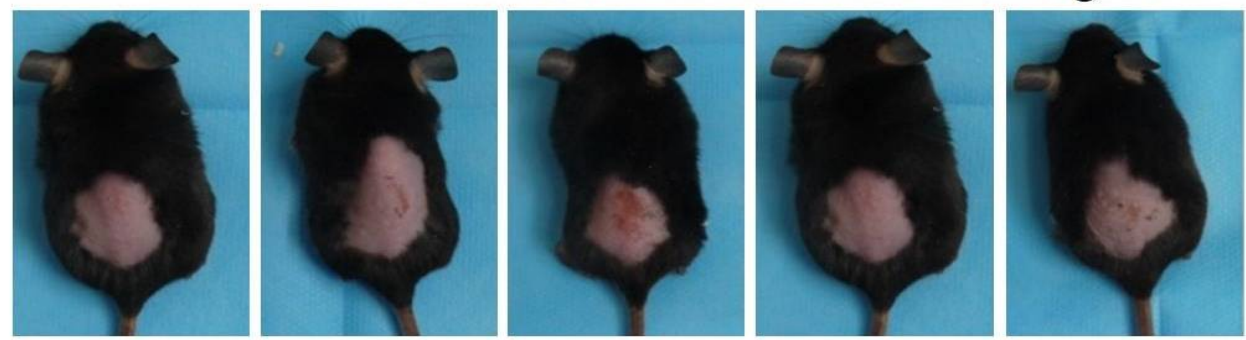

B
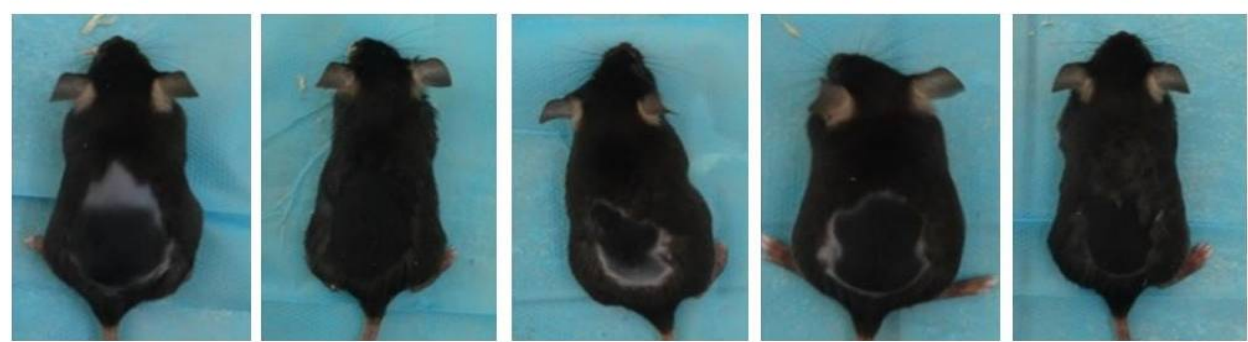

Figure 1: Effects of IPL therapy on hair growth in C57BL/6J mice in vivo. A and B represent 0 and 21 days after depilation, respectively.

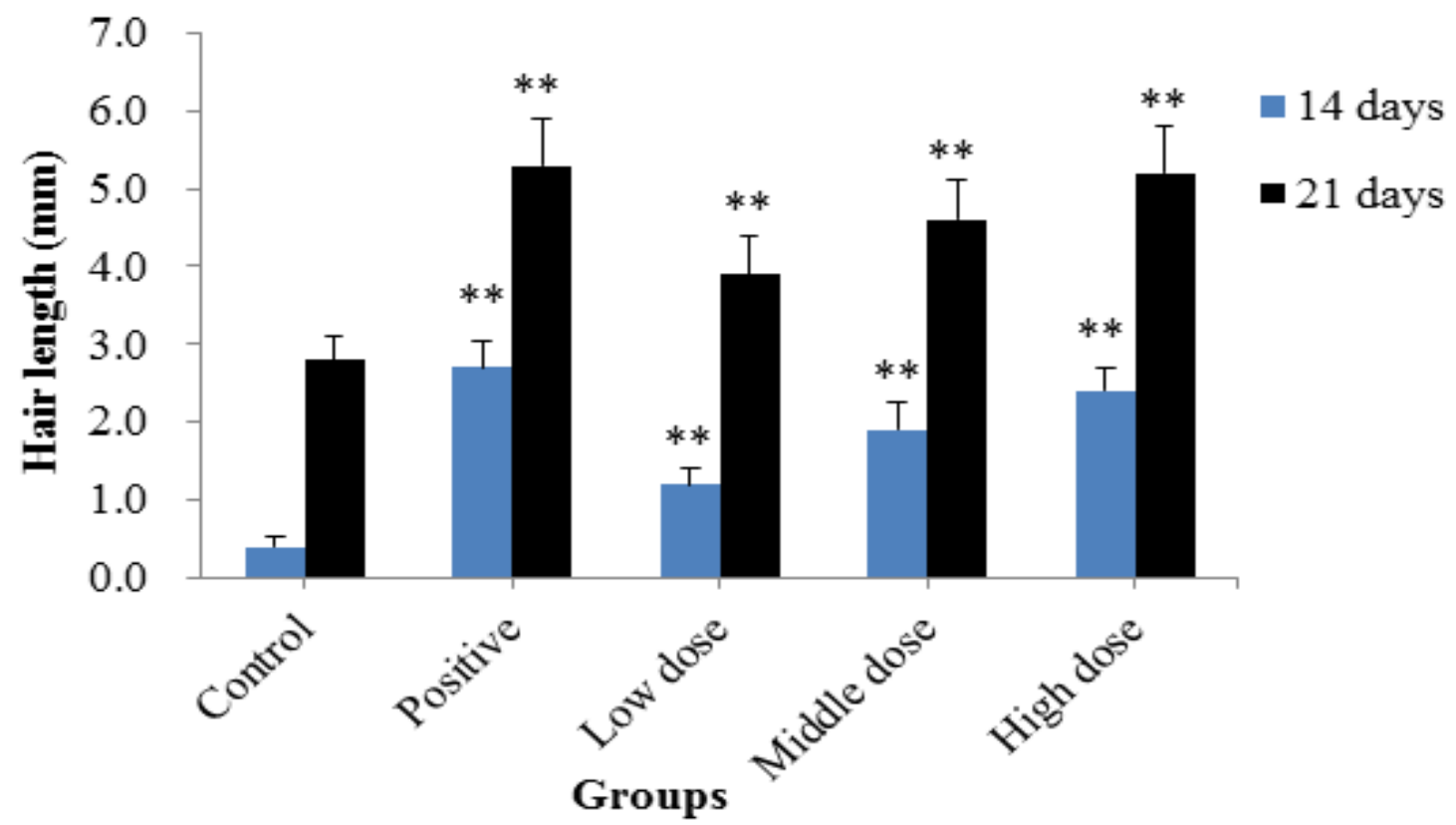

Figure 2: Hair length of C57BL/6J mice at 14 and 21 days after depilation. ${ }^{* *} p<0.01$, versus control group 

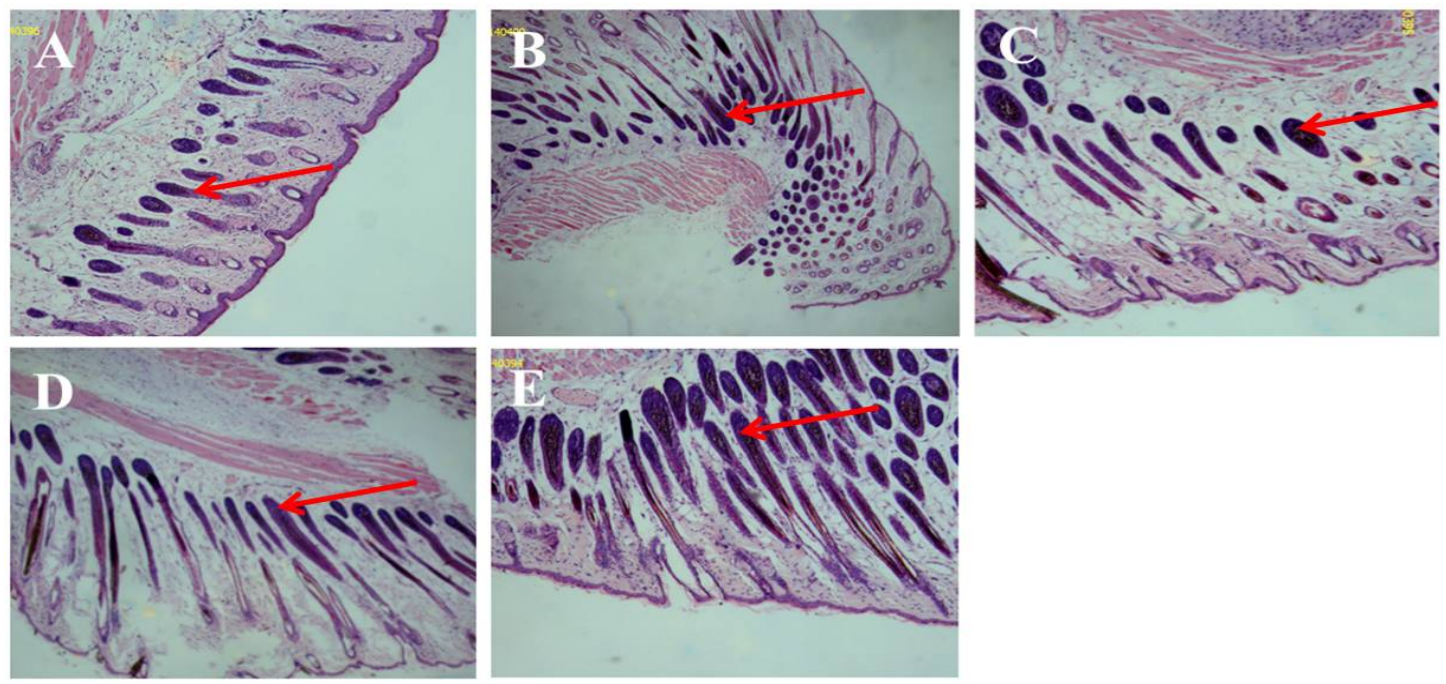

Figure 3: Histopathological examination of the skin of C57BL/6J mice (200x). A, B, C, D, and E represent control, positive, low dose IPL, middle dose IPL and high dose IPL, respectively. The number of hair follicles (arrowhead) was increased in the positive and different IPL groups
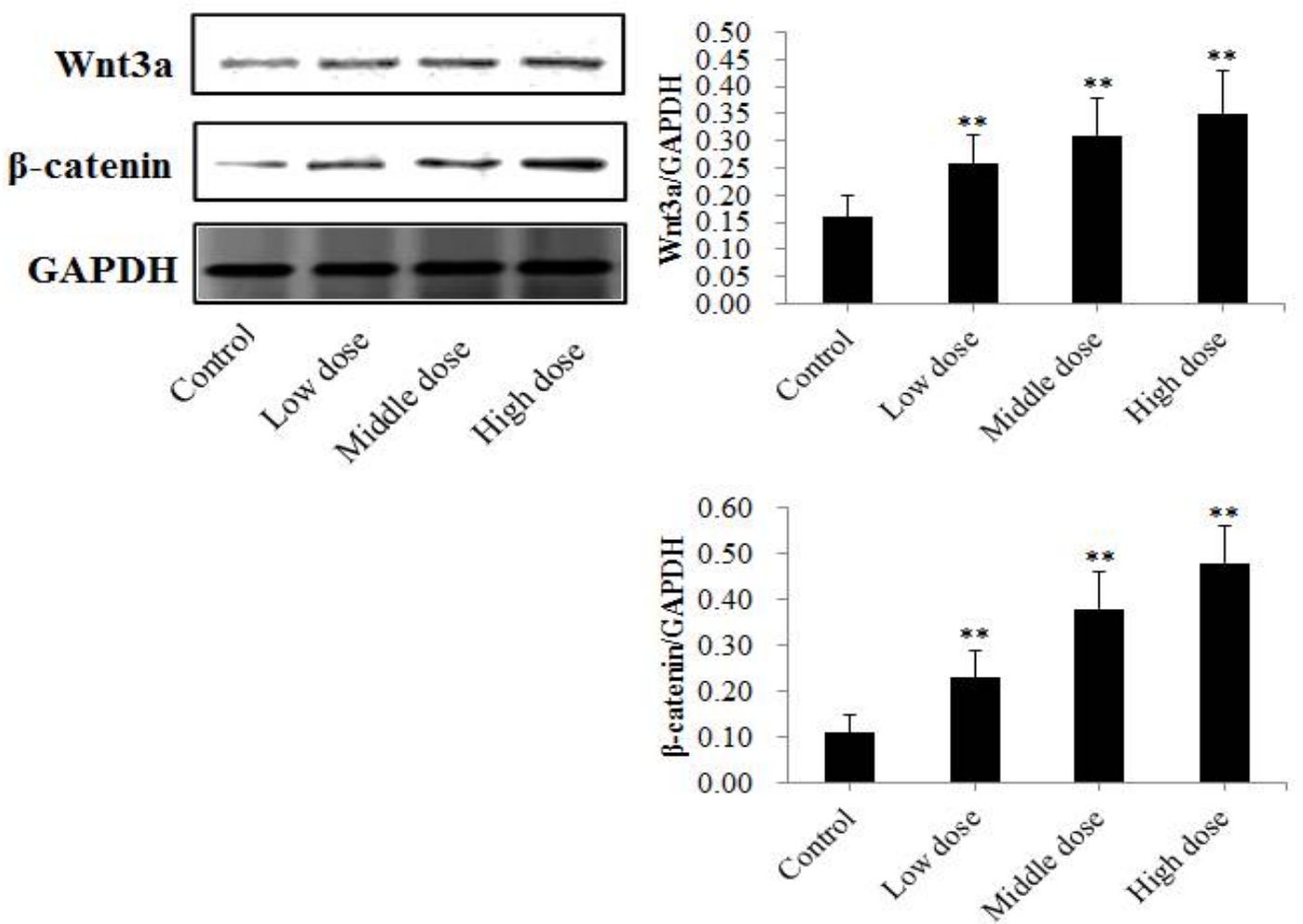

Figure 4: Expressions of WNT3A and $\beta$-catenin in the skin tissue of mice. ${ }^{*} p<0.01$, versus control group

\section{Effect of low-fluence IPL on the development and structure of hair follicles}

To investigate the status of hair follicles, $\mathrm{H}$ \& $\mathrm{E}$ stained skin tissues were observed by optical microscopy. In the representative longitudinal sections, low-fluence IPL irradiation visibly increased the number of hair follicles in mice, compared to the control group after 21 days. Histopathological analysis indicated that lowfluence IPL effectively promoted hair growth in C57BL/6J mice (Figure 3).

\section{$\beta$-Catenin and WNT3A expression in the skin tissue of C57BL/6J mice}

To elucidate the mechanism underlying the induction of the anagen phase in IPL treated groups, western blotting assays were performed. The results revealed that the protein levels of $\beta$ catenin and WNT3A at 21 days after depilation were higher in IPL treated groups, compared to the control group $(p<0.01)$ (Figure 4$)$. 


\section{DISCUSSION}

Androgenetic alopecia is caused by decreased proliferation of follicle epithelia and miniaturization of terminal hairs on the scalp [10]. However, current treatments are not effective for all patients with androgenetic alopecia. Therefore, it is necessary to develop new therapies for hair loss.

Several reports have shown that paradoxical hypertrichosis is a rare and significant side effect of IPL and laser treatments and, occurs after several months within and/or proximal to lasertreated areas [2]. In the present study, the dorsal skin color of mice was darker in IPL-treated group than the control group. After 14 and 21 days, the hair length in IPL treated group was significantly longer than that of the control group, indicating earlier hair follicle induction in these groups. In addition, histopathological analysis revealed that the percentage of hair follicles in anagen stages of IPL treated groups was higher than the control group. These results suggest that the hair growth-promoting activity of IPL may be mediated by early conversion from telogen to anagen and prolonged duration of the anagen phase of hair follicles in C57BL/6J mice.

It is known that the WNT/B-catenin pathway plays an important role in the regulation of the hair cycle, hair growth, and cell proliferation [1113]. Among the WNT/ $\beta$-catenin signaling molecules, $\beta$-catenin and WNT3A are key regulators of hair follicle growth and cycling. Indeed, each has been reported to be the primary initiator of the anagen phase [14].

To elucidate the molecular mechanism by which IPL promote hair growth, the protein levels of $\beta$ catenin and WNT3A in the depilated dorsal skin 21 days after treatment were evaluated by western blotting assays. The results showed that the expression of $\beta$-catenin and Wnt3a in IPL treated mice was up-regulated compared to the control group. These results indicate that the effects of IPL are associated with the development of new hair follicles; and IPL may activate hair follicle development through the WNT/ $\beta$-catenin pathway. Specifically, it is possible that IPL stimulated the hair follicles from telogen to anagen phase and prolongs the duration of the anagen phase. Thus, low-fluence IPL may be a potential and convenient treatment option for hair regrowth.

However, this study has several limitations. First, the results obtained with mice may not translate to identical effects in human skin. Second, the present study did not evaluate signaling pathways beyond the canonical WNT10B/ $\beta$ catenin pathway.

\section{CONCLUSION}

This study is the first to identify the hair growth promoting activity of low-fluence IPL treatment via activation of the WNT/ $\beta$-catenin pathway. These findings suggest that low-fluence IPL treatment is a potential novel modality for hair loss therapy.

\section{DECLARATIONS}

\section{Acknowledgement}

This study was supported by Natural Science Foundation of Guangdong Province (nos. 2015A030311001 and 2017A030310120), Science and Technology Planning Project of Guangzhou (no. 201508020262), National Natural Science Foundation of China (nos. 81772104 and 81701929), Special Foundation for Economic and Technological Development in Longgang District of Shenzhen (no. 2017040518323340).

\section{Conflict of interest}

No conflict of interest is associated with this work.

\section{Contribution of authors}

We declare that this work was done by the authors named in this article and all liabilities pertaining to claims relating to the content of this article will be borne by the authors. Zhiqi Hu conceived and designed the study, Zhexiang Fan and Jin Wang collected and analyzed the data, Yong Miao and Zhexiang Fan wrote the manuscript. All authors have read and approved the manuscript for publication.

\section{REFERENCES}

1. Rogers NE, Avram MR. Medical treatments for male and female pattern hair loss. J Am Acad Dermatol 2008; 59: 547-566.

2. Desai S, Mahmoud BH, Bhatia AC, Hamzavi IH. Paradoxical hypertrichosis after laser therapy: a review. Dermatol Surg 2010; 36(3): 291-298.

3. Moreno-Arias G, Castelo-Branco C, Ferrando J. Paradoxical effect after IPL photoepilation. Dermatol Surg 2002; 28(11): 1013-1016.

4. Willey A, Torrontegui J, Azpiazu J, Landa N. Hair stimulation following laser and intense pulsed light 
photo-epilation: review of 543 cases and ways to manage it. Lasers Surg Med. 2007; 39(4): 297-301.

5. Bernstein EF. Hair growth induced by diode laser treatment. Dermatol Surg 2005; 31(5): 584-586.

6. Ouchi $N$, Shibata $R$, Walsh K. AMP-activated protein kinase signaling stimulates VEGF expression and angiogenesis in skeletal muscle. Circ Res 2005; 96: 838-846

7. Yano K, Brown LF, Detmar M. Control of hair growth and follicle size by VEGF-mediated angiogenesis. J Clin Invest 2001; 107: 409-417.

8. "Principles of Laboratory Animal Care" (NIH publication no. 85-23, revised 1985). Available from: http://grants 1.nih.gov/grants/olaw/references/phspol.htm

9. Muller-Rover $S$, Handjiski $B$, van der Veen $C$, Eichmuller S, Foitzik K, McKay IA, Stenn KS, Paus R. A comprehensive guide for the accurate classification of murine hair follicles in distinct hair cycle stages. J Investig Dermatol 2001; 117(1): 3-15

10. Olsen EA, Messenger AG, Shapiro J, Bergfeld WF, Hordinsky MK, Roberts JL, Stough D, Washenik K, Whiting DA. Evaluation and treatment of male and female pattern hair loss. J Am Acad Dermatol 2005; 52(2): 301-311.

11. Clevers $H$. Wnt/beta-catenin signaling in development and disease. Cell 2006; 127: 469-480.

12. Tsai SY, Sennett R, Rezza A, Clavel C, Grisanti L, Zemla $R$, Najam S, Rendla M. Wnt/beta-catenin signaling in dermal condensates is required for hair follicle formation. Dev Biol 2014; 385(2): 179-188.

13. Wodarz A, Nusse R. Mechanisms of Wnt signaling in development. Ann Rev Cell Dev Biol 1998; 14: 59-88.

14. Millar SE. Molecular mechanisms regulating hair follicle development. J Invest Dermatol 2002; 118(2): 216-225. 